\title{
ARTíCULO \\ Patrones estacionales y espaciales de la diversidad de moluscos intermareales de bahía Róbalo, canal Beagle, Reserva de la Biosfera Cabo de Hornos, Chile
}

Seasonal and spatial patterns of intertidal molluscs diversity of Róbalo Bay, Beagle Channel, Biosphere Reserve Cape Horn, Chile

\author{
Jaime Ojeda ${ }^{1,2,3}$, Sebastián Rosenfeld ${ }^{1,3}$, Johanna Marambio ${ }^{1,3}$, \\ Ricardo $\operatorname{Rozzi}^{2,3,4}$ y Andrés Mansilla ${ }^{1,2,3}$
}

\begin{abstract}
${ }^{1}$ Laboratorio de Macroalgas Antárticas y Subantárticas (LMAS), Universidad de Magallanes, Casilla 113-D, Punta Arenas, Chile.jaimeojedavillarroel@yahoo.es

${ }^{2}$ Parque Etnobotánico Omora, Universidad de Magallanes, Teniente Muñoz 396, Puerto Williams, Chile ${ }^{3}$ Instituto de Ecología \& Biodiversidad (IEB), Facultad de Ciencias, Universidad de Chile, Casilla 653, Santiago, Chile ${ }^{4}$ Sub-Antarctic Biocultural Conservation Program, Department of Philosophy and Religion Studies and Department of Biological Sciences, University of North Texas, Denton TX, USA
\end{abstract}

\begin{abstract}
Chilean sub-Antarctic Channels region is one of the most diverse regions in molluscs species of the South Pacific Ocean in South America. This region exhibit intertidal zones with a high environmental heterogeneity at a local scale, including seasonal changes in photoperiod, rocky substrate diversity, salinity and temperature gradients. This study characterized molluscs assemblages in Róbalo bay 54 $55^{\prime}$ 'S (Beagle Channel), identifying spatial-temporal changes in assemblage composition, species richness, abundance, functional groups, and diversity of molluscs species. We registered 34 species comprising 4 functional groups, being mobile herbivores the most diverse group (21 species). The most abundant molluscs were Mytilus edulis platensis and Perumytilus purpuratus. Considering the mobile species only, the most abundant were Laevilitorina caliginosa and Siphonaria lessonii. In seasonal terms, species richness and abundance of mobile herbivores showed an increase during the austral summer. While the abundance of $M$. edulis platensis and mobile carnivores remained stable during the seasons. Regarding vertical zonation within the intertidal, we found that $\mathrm{L}$. caliginosa is mainly associated with high intertidal, Pareuthria plumbea to the mid-zone, and N. deaurata to the low intertidal zone. Róbalo Bay high beta molluscs diversity was associated to the spatial variability of intertidal zones. These changes can be detected in between sites separated only by hundreds of meters, where local environmental heterogeneity likely plays a role structuring ecological assemblages and communities Sub-Antarctic channels.
\end{abstract}

Key words: Sub-Antarctic channels, seasonal changes, rocky intertidal, Naticidae, Nacella

Resumen.- Los canales subantárticos de Chile son una de las regiones con mayor diversidad de moluscos del océano Pacífico en el sur de Sudamérica. Esta región presenta extensas zonas intermareales sujetas a una marcada estacionalidad con cambios en el fotoperiodo y una alta heterogeneidad ambiental, que incluye diversidad de sustratos, gradientes de salinidad y temperatura. El presente estudio caracterizó el ensamble de moluscos de bahía Róbalo 5455'S (canal Beagle) y evaluó los cambios espacio-estacionales de la composición, riqueza y diversidad de moluscos. Se registraron 34 especies de moluscos, y se caracterizaron 4 grupos funcionales, siendo el más diverso los herbívoros móviles (21 especies). Los moluscos más abundantes fueron los filtradores sésiles, Mytilus edulis platensis y Perumytilus purpuratus. Las especies móviles más abundantes fueron Laevilitorina caliginosa y Siphonaria lessonii. En términos estacionales, la riqueza de especies y abundancia de los herbívoros móviles presentaron un aumento durante el verano austral. Mientras que la abundancia de M. edulis platensis y los carnívoros móviles se mantuvieron estables durante las estaciones. Respecto a la zonificación vertical dentro del intermareal, se encontró que L. caliginosa está asociada principalmente al intermareal alto, Pareuthria plumbea a la zona media y N. deaurata al intermareal bajo. En bahía Róbalo se registró una alta diversidad beta de moluscos, asociada a la variabilidad espacial de las zonas intermareales. Estos cambios se pueden detectar entre sitios separados por centenas de metros, donde la heterogeneidad ambiental local probablemente juega un rol ecológico en la estructuración de ensambles y comunidades en canales subantárticos.

Palabras clave: Canales subantárticos, cambios estacionales, intermareal rocoso, Naticidae, Nacella 


\section{INTRODUCCIÓN}

Los moluscos constituyen uno de los principales grupos de organismos que conforman ensambles estructuradores de comunidades intermareales (ver Stephenson \& Stephenson 1949, Lewis 1972). Junto a las macroalgas, aportan la mayor riqueza específica de estos ambientes (e.g., Broitman et al. 2011) y muchos de ellos (e.g., los mitílidos) proveen de sustrato a otras especies (Gutiérrez et al. 2003). También los moluscos constituyen uno de los principales grupos de consumidores tanto carnívoros como herbívoros, modelando la estructura comunitaria del intermareal (e.g., Moreno et al. 1984, Castilla \& Durán 1985). A su vez, son sensibles a cambios ambientales de carácter estocástico o periódico, como cambios estacionales por fotoperiodo o formación de hielo (e.g., Kim 2001, Harley 2002, Scrosati \& Heaven 2007).

Las costas templadas frías del océano Pacífico en el sur de Sudamérica exhiben un patrón de riqueza de especies de moluscos que aumenta hacia latitudes mayores, esto específicamente para taxones como la clase Polyplacophora, subclase Prosobranchia y clase Bivalvia (ver Valdovinos et al. 2003). Uno de los principales ecosistemas costeros de estos ambientes, es el sistema de canales y fiordos del sur de Chile $\left(42^{\circ} \mathrm{S}-56^{\circ} \mathrm{S}\right)$, que presenta varios atributos ecológicos a escala espacial, temporal y biogeográfico, por ejemplo: i) su topografía fue moldeada por la erosión glacial del continente, debido al avance y retroceso de los hielos ocurridos durante el cuaternario (Silva \& Calvete 2002), que en el Último Máximo Glacial cubrieron la mayor parte del territorio entre 23.000 y 19.000 años A.P (Hulton et al. 2002); ii) presenta marcados gradientes oceanográficos asociados a factores como exposición al oleaje, temperatura y salinidad (ver Dayton 1985, Silva \& Calvete 2002); iii) la biota marina y específicamente los moluscos estructuran la denominada unidad biogeográfica Magallánica (ver Stuardo 1964, Camus 2001); iv) dentro del registro fósil de moluscos se plantea que la colonización y posterior diversificación fue producido durante el pleistoceno tardío (Kiel \& Nielsen 2010) y v) existen evidencias de procesos evolutivos de diversificación genética reciente para gastrópodos, como el género Nacella (González-Wevar et al. 2011).

En los canales subantárticos destacan los estudios malacológicos taxonómicos realizados por expediciones científicas del siglo XIX y comienzos del siglo XX, como describen detalladamente Reid \& Osorio (2000). Hasta el presente se han registrado al menos 437 especies de moluscos para esta región de canales (Valdovinos 1999).
En contraste con los estudios taxonómicos, la caracterización ecológica de las comunidades intermareales y específicamente de los moluscos ha sido poco atendida. Por ejemplo, destacan los trabajos desarrollados en el área de la paleo-ecología, donde análisis tafonómicos documentan alrededor de 45 especies de moluscos (ver Cárdenas \& Gordillo 2009). Otros estudios de la composición y estructura intermareal provienen de la zona del estrecho de Magallanes que registran al menos unas 26 especies de moluscos (e.g., Mutschke et al. 1998, Ríos \& Mutschke 1999, Aldea \& Rosenfeld 2011). Considerando los patrones descritos de alta riqueza de especies para la región biogeográfica Magallánica de canales (ver Linse 1999, Valdovinos 1999), la riqueza de especies de moluscos intermareales a escala local debería aumentar. No obstante, aún es necesario incrementar el número de estudios en zonas intermareales subantárticas, ya que el mayor aporte proviene principalmente por cruceros científicos focalizados en estudios pelágicos, del bentos profundo y la zona submareal.

En latitudes altas, la estacionalidad es otro factor fundamental para entender la dinámica del intermareal. El fotoperiodo y la intensidad de la radiación solar presentan marcados cambios estacionales que determinan variaciones en la diversidad y abundancia de macroalgas (e.g., Gunnarsson \& Ingólfsson 1995), que podrían incidir en las abundancias y diversidad de moluscos. Harley (2002) determinó una influencia relativa sobre la disminución de algas filamentosas en invierno, por efecto de la disminución de luz, fenómeno que causa una menor densidad de gastrópodos herbívoros e incluso puede tener efectos en la biomasa de los individuos. Para los moluscos sésiles en canales subantárticos se ha documentado que las matrices de Mytilus edulis platensis (d'Orbigny, 1846) no presentan cambios estacionales en la abundancia (Langley et al. 1980). Sin embargo, otros antecedentes indican cambios significativos en biomasa y riqueza de macroalgas intermareales entre invierno y verano (Ojeda 2012), los cuales que podrían determinar cambios estacionales en la densidad de herbívoros móviles. Cabe destacar, que en la zona de canales y fiordos subantárticos las investigaciones de las comunidades intermareales han estado sesgadas principalmente a evaluaciones estivales, careciendo de información invernal o que evalúen una dinámica estacional. Estudios de variaciones estacionales son esenciales para comprender la ecología de las comunidades intermareales de Magallanes e investigar las variaciones a escala planetaria puesto que el clima de la eco-región subantártica 
de Magallanes contrasta marcadamente con el clima de zonas templadas frías y subárticas del Hemisferio Norte (ver Rozzi et al. 2012).

Respecto a los patrones de distribución espacial, los estudios en la zona de canales y fiordos subantárticos del sur de Chile, también pueden tener una relevancia global. La clásica caracterización de los patrones de zonación de las especies en costas rocosas y su interacción con los niveles intermareales proponen una universalidad en la estructura vertical espacial del intermareal (Stephenson \& Stephenson 1949). Esta propuesta parece tener una aplicabilidad general en costas expuestas al oleaje, pero no parece ser consistente en costas protegidas al oleaje (Raffaelli \& Hawkins 1996). Una de las principales razones de estos cambios se atribuye a la heterogeneidad ambiental (Foster 1990). Estudios referentes a los patrones de zonación vertical en la zona de canales y fiordos, muestran que no existe un patrón general de zonación (Benedetti-Cecchi \& Cinelli 1997). Estos cambios fueron identificados principalmente para especies sésiles conspicuas, como M. edulis platensis, que varía su cobertura considerablemente entre un sitio y otro (ver Benedetti-Cecchi \& Cinelli 1997). No obstante, aún es necesario investigar la composición específica de las especies en su distribución vertical y horizontal, para evaluar las variaciones de la diversidad alfa y beta asociadas a la alta heterogeneidad de tipo de hábitats y micro-hábitats intermareales. El estudio de un ensamble diverso y conocido como los moluscos puede entregar información sobre la dominancia, rareza y recambio de especies en asociación con la heterogeneidad ambiental. Este estudio requiere considerar tipos de muestras extractivas que analizan componentes que habitan bajo y sobre las rocas, puesto que se ha documentado que en zonas intermareales polares la riqueza de taxones puede aumentar bajo los bolones rocosos (ver Waller 2008).

Los objetivos del presente trabajo son: i) caracterizar el ensamble de moluscos presentes en una bahía con alta heterogeneidad ambiental que se ubica en la costa sur del canal de Beagle, bahía Róbalo; ii) investigar variaciones estacionales de composición, riqueza y abundancia de los moluscos, considerando grupos funcionales como herbívoros móviles; e iii) investigar variaciones espaciales en la composición del ensamble de moluscos asociadas al gradiente vertical del intermareal, y a la heterogeneidad ambiental en el gradiente horizontal de la bahía. La caracterización de los probables cambios estacionales-espaciales del ensamble de moluscos, complementa los estudios realizados anteriormente en canales subantárticos, que se han centrado en períodos breves de muestreo, principalmente durante la estación estival.

\section{MATERIALES Y MÉTODOS}

\section{Área de ESTUdio}

El presente estudio se realizó entre el 2008-2009 en bahía

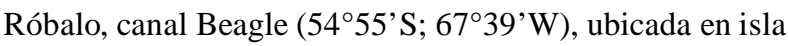
Navarino (Fig. 1). Este sitio se encuentra próximo al Parque Etnobotánico Omora perteneciente a la Red Chilena de Sitios de Estudios Socio-Ecológicos a Largo Plazo (Rozzi et al. 2012). El Parque Omora es también el centro de investigaciones interdisciplinarias de la Reserva de Biosfera Cabo de Hornos, que integra ecosistemas terrestres y marinos (Rozzi et al. 2007). En la zona del canal Beagle y específicamente en las cercanías de bahía Róbalo las variables de luminosidad como el fotoperiodo y la Radiación Fotosintéticamente Activa (Luz PAR) presentan cambios estacionales. Por ejemplo, durante el invierno austral (junio) se detectan medidas promedio de $8 \mathrm{~h}$ de luz con un promedio mensual de luz PAR de 23,2 $\mathrm{Wm}^{-2}$, mientras que en el verano austral (diciembre) el promedio cambia a $14 \mathrm{~h}$ luz con un promedio mensual de luz PAR de 164,7 $\mathrm{Wm}^{-2}$ (CEAZA-MET 2010 ${ }^{1}$ ). La temperatura superficial del mar durante invierno presenta valores mínimos de $4,7^{\circ} \mathrm{C}$ y durante verano máximos de $10,9^{\circ} \mathrm{C}$ (datos desde sensor de temperatura bahía Róbalo 2011, Andrés Mansilla, datos sin publicar). La bahía Róbalo posee una longitud aproximada de $6,5 \mathrm{~km}$ y el intermareal presenta una alta heterogeneidad ambiental local en un gradiente horizontal del intermareal, por ejemplo: i) desemboca el río Róbalo al interior de la bahía; ii) Se caracteriza por presentar diferentes sustratos rocosos; y iii) presenta diferentes grados de exposición al oleaje, debido principalmente a la incidencia del viento predominante durante el año con dirección Oeste-Este en la zona del canal Beagle (ver Santana et al. 2006).

Debido a la heterogeneidad en el gradiente horizontal y específicamente en los diferentes tipos de sustratos rocosos (guijarros, canto rodado pequeño, canto rodado grande y terraza), el intermareal de la bahía Róbalo se clasificó en 6 sectores, cada uno con una extensión

${ }^{1}$ Red Meteorológica CEAZA-MET. 2010. Red de sensores meteorológicos, descarga de datos del sensor próximo al Parque Etnobotánico Omora, Puerto Williams, Chile. <http://www.ceazamet.cl> 
aproximada de $150 \mathrm{~m}$ en paralelo a la línea de costa, con el fin de investigar posibles cambios espaciales de tipo horizontal en composición, riqueza y abundancia de moluscos (ver Fig. 1). Cada sector se caracterizó por el tipo de sustrato predominante de forma cualitativa y cuantitativa (clasificación de sustratos según Raffaelli \& Hawkins [1996]). La clasificación cuantitativa se realizó mediante 100 puntos al azar dentro de cada sector, en cada punto se midió el tamaño del diámetro de la partícula rocosa (ver Harrelson et al. 1994) (Tabla 1). También como indicador a la exposición al oleaje de cada sector intermareal, se evaluó el grado de exposición al viento Oeste. Para ello, se realizaron 4 mediciones para cada sector con un anemómetro Kestrel 2500, durante octubre (2008), obteniendo la velocidad promedio en $\mathrm{m} \mathrm{s}^{-1}$ del viento en cada sector intermareal.

\section{DISEÑO DE MUESTREO, IDENTIFICACIÓN Y CUANTIFICACIÓN DE MOLUSCOS}

Los muestreos de moluscos estacionales fueron realizados entre el 20-25 de mayo 2008 (otoño), 10-15 de agosto 2008 (invierno), 20-25 de octubre 2008 (primavera) y 15-20 de enero 2009 (verano). En cada sector intermareal se categorizaron 3 niveles asociado a la altura vertical del intermareal (alto, medio y bajo), estos definidos mediante el protocolo descrito por Benedetti-Cecchi \& Cinelli (1997). Es decir, se revisaron valores y fechas de las mareas más altas y bajas predichas por las tablas de marea publicadas para la localidad de Puerto Williams (ver SHOA 2008²). Con esta información se planificó el registro in situ del límite espacial de las mareas más bajas y altas en bahía Róbalo, en condiciones de mar calma. El muestreo se realizó en los 2 extremos (niveles altos y bajos), y en

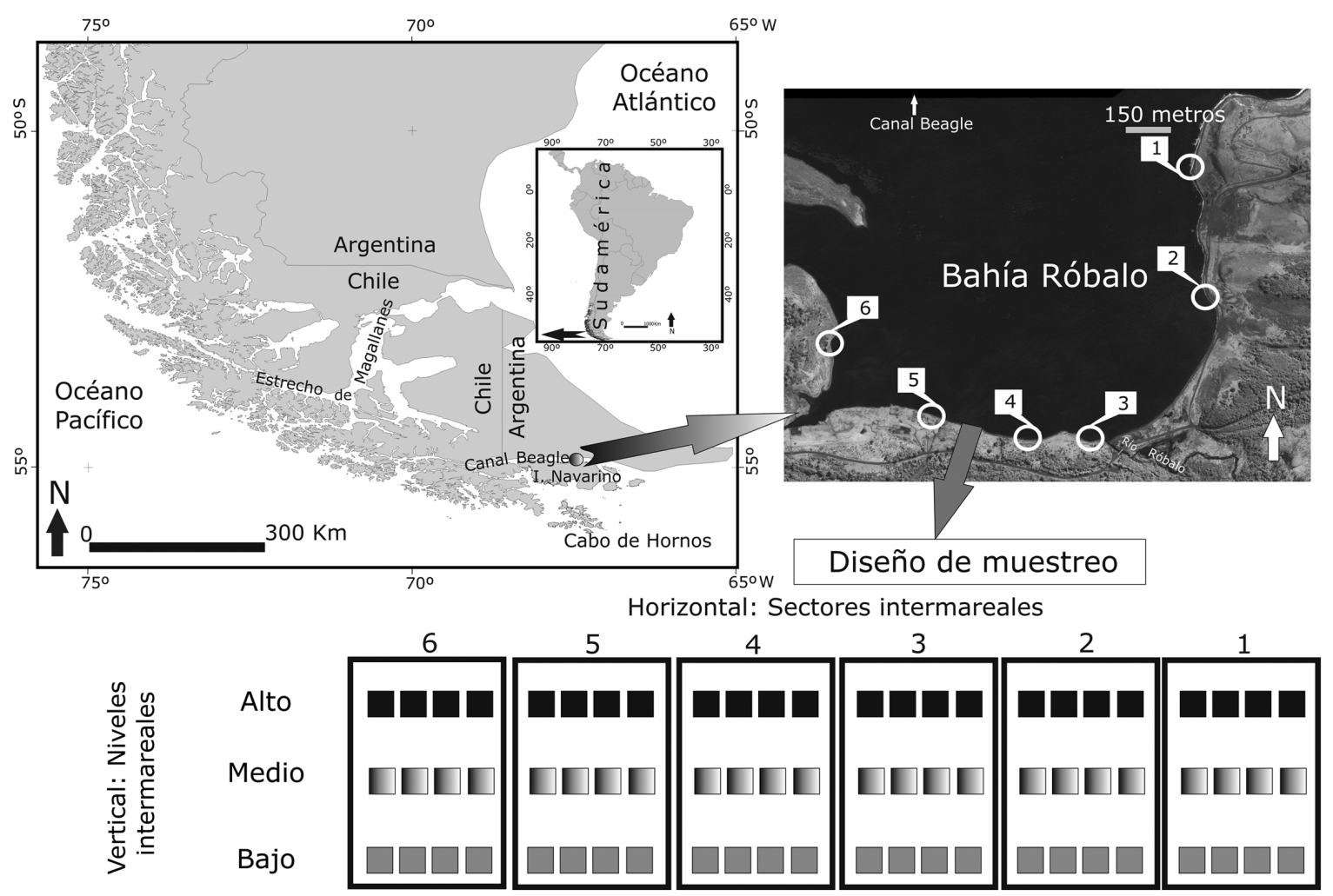

Figura 1. Sitio de estudio en bahía Róbalo, canal Beagle ( $\left.54^{\circ} 55^{\prime} \mathrm{S} ; 67^{\circ} 39^{\prime} \mathrm{W}\right)$, isla Navarino, Reserva de Biosfera Cabo de Hornos, Chile. A escala espacial horizontal se distinguieron 6 sectores intermareales y a escala vertical se definieron 3 alturas intermareales: alto, medio y bajo /

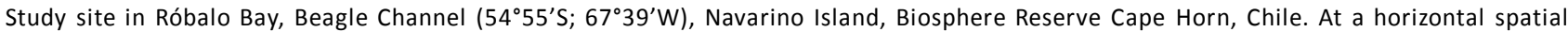
scale, we distinguished 6 sectors, and we defined 3 intertidal heights at a vertical scale: high, medium and low

${ }^{2}$ SHOA. 2008. Servicio Hidrográfico y Oceanográfico de la Armada de Chile. Disponibilidad de datos diarios de mareas de sicigia y cuadratura para Puerto Williams, isla Navarino, Chile. <http://www.shoa.cl> 
Tabla 1. Caracterización de sectores intermareales de bahía Róbalo, canal Beagle (5455’S; 67³9’ W). Se indica la clasificación cualitativa de sustrato predominante (según Raffaelli \& Hawkins 1996), y cuantitativa de sustrato rocoso (según Harrelson et al. 1994). Se detalla la exposición y la velocidad al viento Oeste para cada sector intermareal con 4 mediciones puntuales durante octubre del 2008 / Róbalo Bay (Beagle Channel, 5455'S; 67³9' W) intertidal areas characterization, the table shows the qualitative classification of dominating substrate (according to Raffaelli \& Hawkins 1996), and quantitative classification of rocky substrates (according to Harrelson et al. 1994). It shows wind exposure and west windspeed for each intertidal sector measured in 4 point during October 2008

\begin{tabular}{|c|c|c|c|c|c|c|}
\hline & \multicolumn{6}{|c|}{ Sectores intermareales } \\
\hline & 1 & 2 & 3 & 4 & 5 & 6 \\
\hline Categoría cualitativa & $\begin{array}{l}\text { Canto rodado } \\
\text { grande }\end{array}$ & $\begin{array}{l}\text { Canto rodado } \\
\text { pequeño }\end{array}$ & $\begin{array}{l}\text { Canto rodado } \\
\text { pequeño }\end{array}$ & $\begin{array}{l}\text { Canto rodado } \\
\text { grande }\end{array}$ & Terraza & $\begin{array}{c}\text { Canto rodado } \\
\text { pequeño }\end{array}$ \\
\hline \multicolumn{7}{|l|}{ Categoría cuantitativa } \\
\hline$\%$ Arena & 0 & 2 & 45 & 0 & 0 & 1 \\
\hline$\%$ Guijarro & 4 & 6 & 12 & 0 & 0 & 5 \\
\hline$\%$ Canto rodado pequeño & 29 & 47 & 42 & 5 & 0 & 83 \\
\hline$\%$ Canto rodado grande & 65 & 45 & 1 & 95 & 0 & 11 \\
\hline$\%$ Terraza & 2 & 0 & 0 & 0 & 100 & 0 \\
\hline Velocidad del viento $(\mathrm{m} / \mathrm{s})$ & $9,9 \pm 0,4$ & $10,4 \pm 0,8$ & $4,8 \pm 0,8$ & $4,8 \pm 0,5$ & $5,8 \pm 0,6$ & $6 \pm 0,4$ \\
\hline Exposición al viento Oeste & Expuesto & Expuesto & Protegido & Protegido & Protegido & Protegido \\
\hline
\end{tabular}

distancias equidistantes los niveles intermareales medios. En cada nivel intermareal se tomaron 4 cuadrantes al azar de $25 \times 25 \mathrm{~cm}$ (área de $625 \mathrm{~cm}^{2}$ ). Por lo tanto, el diseño de muestreo fue de 4 (estaciones) x 6 (sectores) x 3 (niveles intermareales) $\times 4$ cuadrantes $=288$ muestras (Fig. 1). En cada cuadrante la colecta de moluscos se realizó bajo y sobre las rocas intermareales, estos fueron almacenados en formalina al $5 \%$ para la identificación a nivel de familia, especie y grupo funcional. Para esta última categoría se consideraron los hábitos de movilidad (sésil o móvil) y hábitos tróficos (e.g., filtrador, herbívoro o carnívoro). La clasificación de los hábitos tróficos se realizó utilizando la literatura existente para cada grupo taxonómico (e.g., Camus et al. 2009, 2013). También se clasificó de forma descriptiva el tamaño de los componentes del ensamble, es decir, las especies identificadas con un tamaño de adulto $<5 \mathrm{~mm}$ fueron consideradas micromoluscos (ver Geiger et al. 2007), mientras que tamaños superiores se consideraron macromoluscos $(>5 \mathrm{~mm})$. Con el fin de establecer listas de especies más completas para bahía Róbalo, se consideró también las especies de moluscos que no ocurrieron dentro de los cuadrantes del protocolo de muestreo. Estos registros, sin embargo, no fueron incluidos en los análisis estadísticos comparativos de ningún tipo. La abundancia se determinó mediante el número de individuos/especie/cuadrante en $625 \mathrm{~cm}^{2}$. Para la identificación de moluscos se realizaron observaciones macroscópicas utilizando un estéreo-microscopio Olympus ${ }^{\circledR}$ SZ61, con el apoyo de bibliografía taxonómica malacológica para la identificación a los niveles de mayor resolución taxonómica. Por ejemplo, para la clase Polyplacophora, Gastropoda y Bivalvia se utilizó: Powell (1951), Dell (1964), Reid \& Osorio (2000), Linse (2002), Sirenko (2006), Ojeda et al. (2010), Aldea \& Rosenfeld (2011); También se uso información desde trabajos clásicos como: Rochebrune \& Mabille (1889) y Strebel (1905). Para taxones específicos, se utilizó literatura especializada como: Chitonidae (Schwabe et al. 2006), Nacellidae (Aranzamendi et al. 2009, González-Wevar et al. 2011), Eatoniellidae (Ponder \& Worsfold 1994), Naticidae (Pastorino 2005), Diaphanidae (Dell 1990, Rosenfeld \& Aldea 2011), Onchidiidae (Rosenfeld \& Aldea 2010), Neoleptonidae (Zelaya \& Ituarte 2004) y Pectinidae (Walozeck 1984).

\section{ANÁLISIS DE DATOS}

La riqueza y abundancia estacional de moluscos de bahía Róbalo fue determinada mediante el número de individuos mensual ( $\mathrm{N}=72$ muestras $)$ y anual $(\mathrm{N}=288$ muestras $)$ por especie. También se determinó la frecuencia de ocurrencia anual por especie (Fr), el promedio de la riqueza y el número de individuos para cada estación de muestreo dentro de la bahía. También se determinó la riqueza esperada por estación. A su vez, se evaluó teóricamente si el esfuerzo de muestreo en cada estación, estimó el total de las especies asociadas al intermareal de bahía Róbalo. Para ambos casos, se utilizó el modelo exponencial negativo de acumulación de especies, diseñado para 
estimar la riqueza esperada dependiendo del número de muestras (Soberón \& Llorente 1993). Este modelo se utiliza para zonas de muestreo pequeñas o para grupos taxonómicos bien conocidos (Jiménez-Valverde \& Hortal 2003). Para la elaboración de las curvas de acumulación de especies, primero se aleatorizó el total de muestras para cada estación. Posteriormente, se utilizó un ajuste no lineal con método Simplex y Quasi-Newton del paquete estadístico STATISTICA 7 (Statsoft 1999³), esto para la obtención de los coeficientes del modelo, es decir $a$ y $b$. Finalmente, se determinó la riqueza máxima esperable para el modelo que se obtiene mediante la división de estos coeficientes $(a / b)$ (Soberón \& Llorente 1993).

Para determinar los posibles cambios del ensamble de moluscos se realizó un diseño anidado, considerando como fuentes de variación a: i) estación, ii) sector y iii) nivel intermareal. El análisis se realizó para variables univariadas y multivariadas mediante el análisis de permutaciones PERMANOVA (Anderson 2005). Para el caso de los datos univariados se usaron variables como la riqueza de especies, abundancia de individuos y las especies con mayor frecuencia de ocurrencia en los cuadrantes. Para ello, se calculó la distancia de disimilaridad de Bray-Curtis entre pares de observaciones, los datos fueron transformados a raíz cuarta y se utilizaron 999 permutaciones sin restricciones de datos (Anderson 2001). El análisis de agrupaciones multivariantes fue realizado mediante la incorporación de diferentes especies en un grupo funcional, considerando a los filtradores sésiles (F-S; 3 especies), herbívoros móviles (H-M; 11 especies) y carnívoros móviles (C-M; 5 especies). Para este análisis multivariado de PERMANOVA, se excluyeron las especies que tuvieran una ocurrencia menor a 2 muestras, posteriormente se calculó la distancia de disimilaridad de Bray-Curtis entre pares de observaciones, los datos fueron transformados a raíz cuarta (para reducir la escala entre variables) y se usaron 999 permutaciones sin restricciones de datos. Finalmente, para cada efecto significativo se realizó un test $a$ posteriori entre pares de grupos (pair-wise), que determina un $t$-estadístico (Anderson 2005). Todos los análisis de PERMANOVA fueron desarrollados con el programa FORTRAN (Anderson 2005). Para visualizar los cambios significativos de los grupos funcionales entre las diferentes estaciones, se realizó el análisis de escalamiento multidimensional MDS, basado en la matriz de distancia de disimilaridad de Bray-Curtis (Kruskal \& Wish 1978).
La caracterización de la diversidad alfa por sectores intermareales se evaluó mediante el índice de diversidad ecológica absoluta de la serie de Hill N(1), que corresponde al antilogaritmo del índice de Shannon y Weaver ( $\left.\mathrm{H}^{\prime}\right)$, es decir, $\mathrm{N}(1)=\mathrm{e}^{\mathrm{H}}$ donde 'e' es la base de los logaritmos naturales $(2,718)$, este índice expresa la equidad directamente en unidades biológicas (especies) (Hill 1973). Para la diversidad beta $(\beta)$, se consideró la definición del reemplazo espacial en la identidad de las especies entre 2 o más áreas, es decir, una medida de diferencia en la composición entre 2 o más ensambles locales (Ver Koleff 2005). Para medir la diversidad beta se consideraron los datos de presencia y ausencia de especies de moluscos para cada sector intermareal durante todo el periodo de estudio. De esta manera, se calculó índice propuesto por Whittaker (1972): $\mathrm{DB}=(\mathrm{S} / \alpha)-1$, en donde, $\mathrm{S}_{\mathrm{c}}$ es la riqueza de especies registrada en un conjunto de sectores y $\alpha=$ número promedio de especies en los sectores intermareales. Así este índice fluctúa entre cero (cuando las muestras fueron absolutamente idénticas en su composición específica) y uno (las muestras fueron absolutamente diferentes en su composición específica) (ver Rau et al. 1998).

La caracterización del ensamble de moluscos en cada nivel o altura intermareal (i.e., alto, medio y bajo), se realizó mediante la contribución de especies a cada grupo funcional por nivel intermareal. Primero, se determinó el porcentaje de contribución SIMPER (ver Clarke 1993), los datos fueron transformados a raíz cuarta, el porcentaje de corte tuvo un límite de $90 \%$ de contribución al grupo funcional. Segundo, para las especies con mayor contribución se determinó la frecuencia de ocurrencia y el número total de individuos por nivel intermareal. Tercero, con el análisis SIMPER se evaluó el promedio de la disimilaridad entre niveles intermareales dependiendo de cada grupo funcional. Los datos analizados mediante SIMPER fueron testeados en el programa PRIMER 5 (PRIMER-E, Ltd. Plymouth, UK).

\section{Resultados}

\section{Caracterización del ensamble de moluscos}

La riqueza de moluscos durante todo el periodo de estudio fue de 34 especies, con un total de 32.022 individuos muestreados. Entre ellas, 9 especies se detectaron sólo fuera de los cuadrantes y 25 especies dentro de los cuadrantes (Tabla 2). Las 34 especies pertenecieron a 28

${ }^{3}$ Statsoft. 1999. Statistica for Windows [Computer program manual]. StatSoft, Inc., Tulsa. <http://www.statsoft.com> 
géneros diferentes e incluyeron 22 familias (Tabla 2). La clase Gastropoda presentó la mayor cantidad de especies con un $61,8 \%$, seguido por la clase Bivalvia $(20,6 \%)$ y Polyplacophora $(17,6 \%)$. Las familias Chitonidae,
Nacellidae y Mytilidae fueron las más diversas, con 3 especies cada una. A nivel de género, Nacella y Tonicia fueron los más diversos con 3 especies cada uno.

Tabla 2. Especies, frecuencia de ocurrencia (Fr) y sumatoria del número de individuos por mes y año de cada especie del ensamble de moluscos en bahía Róbalo. Cada especie fue clasificada dentro de uno de los siguientes grupos funcionales: herbívoro-móvil (H-M), carnívoro-móvil (CM), filtrador-sésil (F-S) e indeterminado (Ind). El signo (+) indica las especies que fueron registradas sólo fuera de los cuadrantes de muestreo / Species, frequency of occurrence (Fr) and sum of the number of individuals per month and year of each species of the molluscs assemblage in Róbalo Bay. Each species was assigned to one of the following functional groups: herbivore-mobile (H-M), carnivore-mobile (C-M), filterersessile (F-S) and indeterminate (Ind). The sign (+) indicates species that were recorded only outside of the sampling quadrants

\begin{tabular}{|c|c|c|c|c|c|c|c|c|}
\hline Especies & Familia & Otoño & Invierno & Primavera & Verano & $\mathrm{N}^{0}$ ind & Fr & $\begin{array}{c}\text { Grupo } \\
\text { funcional }\end{array}$ \\
\hline Tonicia atrata (Sowerby, 1840) & Chitonidae & 0 & 0 & 2 & 1 & 3 & 1,04 & H-M \\
\hline Tonicia chilensis (Frembly, 1827) & Chitonidae & 1 & 0 & 4 & 2 & 7 & 2,43 & H-M \\
\hline Plaxiphora aurata (Spalowsky, 1795) & Mopaliidae & 3 & 2 & 14 & 24 & 43 & 9,72 & H-M \\
\hline Nacella magellanica (Gmelin, 1791) & Nacellidae & 6 & 22 & 33 & 29 & 90 & 14,2 & H-M \\
\hline Nacella deaurata (Gmelin, 1791) & Nacellidae & 4 & 25 & 46 & 90 & 165 & 14,9 & H-M \\
\hline Nacella flammea (Gmelin, 1791) & Nacellidae & 0 & 5 & 5 & 14 & 24 & 2,43 & $\mathrm{H}-\mathrm{M}$ \\
\hline Scurria ceciliana (d Orbigny, 1841) & Lottiidae & 55 & 13 & 109 & 50 & 227 & 13,5 & H-M \\
\hline Fissurella cf. picta (Gmelin, 1791) & Fissurellidae & 0 & 0 & 0 & 3 & 3 & 0,69 & H-M \\
\hline Margarella violacea (King \& Broderip 1832) & Trochidae & 0 & 2 & 1 & 1 & 4 & 1,39 & H-M \\
\hline Eatoniella nigra (d`Orbigny, 1840) & Eatoniellidae & 0 & 0 & 0 & 1 & 1 & 0,35 & H-M \\
\hline Eatoniella picea Ponder \& Worsfold, 1994 & Eatoniellidae & 0 & 0 & 0 & 1 & 1 & 0,35 & $\mathrm{H}-\mathrm{M}$ \\
\hline Laevilitorina caliginosa (Gould, 1849) & Littorinidae & 117 & 179 & 300 & 324 & 920 & 9,72 & H-M \\
\hline Tectonatica impervia (Philippi, 1845) & Naticidae & 0 & 0 & 1 & 0 & 1 & 0,35 & $\mathrm{C}-\mathrm{M}$ \\
\hline Falsilunatia soluta (Gould, 1847) & Naticidae & 0 & 0 & 1 & 2 & 3 & 0,69 & $\mathrm{C}-\mathrm{M}$ \\
\hline Pareuthria plumbea (Philippi, 1844) & Buccinidae & 20 & 35 & 44 & 34 & 133 & 16 & C-M \\
\hline Trophon geversianus (Pallas, 1774) & Muricidae & 1 & 2 & 4 & 2 & 9 & 2,08 & C-M \\
\hline Acanthina monodon (Pallas, 1774) & Muricidae & 0 & 0 & 1 & 1 & 2 & 0,69 & $\mathrm{C}-\mathrm{M}$ \\
\hline Toledonia parelata Dell, 1990 & Diaphanidae & 0 & 0 & 0 & 2 & 2 & 0,35 & Ind \\
\hline Siphonaria lessonii Blainville, 1827 & Siphonariidae & 99 & 181 & 139 & 59 & 478 & 40,3 & H-M \\
\hline Onchidella marginata (Couthouy en Gould, 1852) & Onchidiidae & 36 & 6 & 5 & 22 & 69 & 4,51 & H-M \\
\hline Aulacomya atra (Molina, 1782) & Mytilidae & 34 & 299 & 51 & 84 & 468 & 17,7 & F-S \\
\hline Perumytilus purpuratus (Lamarck, 1819) & Mytilidae & 4640 & 3485 & 3499 & 2405 & 14029 & 68,4 & F-S \\
\hline Mytilus edulis platensis (d'Orbigny, 1846) & Mytilidae & 3594 & 4165 & 4064 & 3118 & 14941 & 83 & F-S \\
\hline Neolepton concentricum (Preston, 1912) & Neoleptonidae & 0 & 25 & 17 & 212 & 254 & 2,08 & F-S \\
\hline Lasaea adansoni (Gmelin, 1791) & Lasaeidae & 1 & 43 & 94 & 7 & 145 & 3,13 & F-S \\
\hline $\begin{array}{l}\text { Ischnochiton stramineus (Sowerby en Broderip \& } \\
\text { Sowerby 1832) }\end{array}$ & Ischnochitonidae & & & & + & & & H-M \\
\hline Tonicia lebruni Rochebrune, 1884 & Chitonidae & & & & + & & & H-M \\
\hline Callochiton puniceus (Gould, 1846) & Callochitonidae & & & & + & & & $\mathrm{H}-\mathrm{M}$ \\
\hline Fissurella oriens Sowerby, 1835 & Fissurellidae & & & & + & & & H-M \\
\hline Margarella expansa (Sowerby, 1838) & Trochidae & & & & + & & & $\mathrm{H}-\mathrm{M}$ \\
\hline Crepipatella dilatata Lamarck, 1822 & Calyptraeidae & & & & + & & & H-M \\
\hline Kerguelenella lateralis (Gould, 1846) & Siphonariidae & & & & + & & & $\mathrm{H}-\mathrm{M}$ \\
\hline Zygochlamys patagonica (King \& Broderip, 1831) & Pectinidae & & & & + & & & F-S \\
\hline Hiatella solida (Sowerby, 1834) & Hiatellidae & & & & + & & & $\mathrm{F}-\mathrm{S}$ \\
\hline $\mathrm{N}=34$ especies & $\mathrm{N}=22$ Familias & & & & & & & \\
\hline $\mathrm{N}^{0}$ total de ind & & 8611 & 8489 & 8434 & 6488 & 32022 & & \\
\hline $\mathrm{N}^{\mathrm{o}}$ ind móviles & & 342 & 472 & 709 & 663 & 2186 & & \\
\hline $\mathrm{N}^{o}$ de Spp. en cuadrantes & & 14 & 16 & 21 & 24 & 25 & & \\
\hline $\mathrm{N}^{\circ}$ de Spp. totales & & 14 & 16 & 21 & 33 & 34 & & \\
\hline
\end{tabular}


En cuanto a la estimación de la riqueza esperada mediante el modelo exponencial negativo, se determinó en otoño un máximo teórico de 13,8 especies y en invierno 16,1 especies (Fig. 2). Estos valores indican que todavía es probable detectar alguna especie más, considerando que la riqueza observada fue de 13 y 16 en otoño e invierno, respectivamente (Fig. 2). Para la estación de primavera y verano la riqueza máxima esperada fue de 17,9 y 21,9 especies, respectivamente (Fig. 2). En cambio la riqueza observada fue de 20 especies en primavera y 24 especies en verano, es decir, se encontraron más especies de lo que el modelo pudo predecir. Estos datos dan cuenta que el esfuerzo de muestreo fue suficiente para poder detectar la riqueza estacional del intermareal rocoso de bahía Róbalo. Incluso al contrastar el mismo valor de la riqueza máxima esperada y la riqueza observa en verano (i.e., 21,9 especies), es posible obtener el mismo valor de riqueza con un total de 52 cuadrantes, lo que probablemente sea suficiente para estimar la riqueza estacional de moluscos.

Respecto a los grupos funcionales, los herbívorosmóviles exhibieron la mayor riqueza de moluscos (21 especies), seguido por los filtradores-sésiles (7 especies) y los carnívoros-móviles (5 especies). El gasterópodo móvil Toledonia parelata se clasificó como indeterminado en términos tróficos (ver Tabla 2). Cabe destacar, que Pareuthria plumbea está catalogado de hábitos

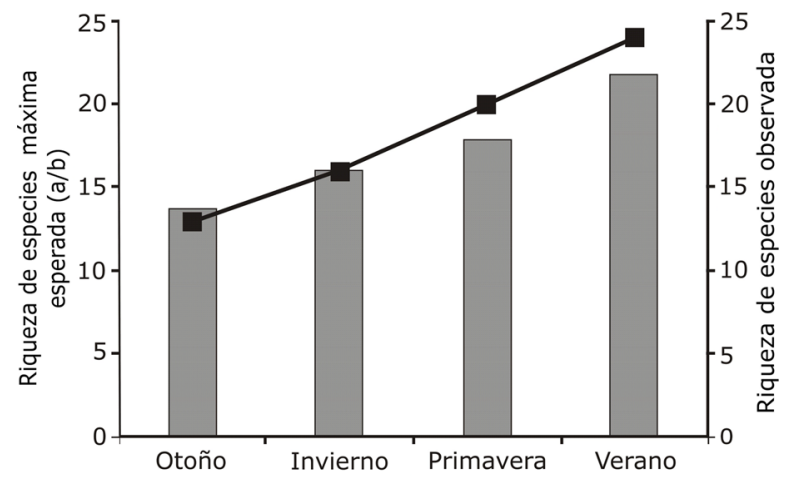

Figura 2. Riqueza de especies máxima esperada para cada estación en barras grises. Esta se estimó con la división de los coeficientes (a/b) del modelo exponencial negativo de acumulación de especies. La riqueza observada (linea negra), se estimó con el total de especies observadas en 72 cuadrantes para cada estación / The maximum species richness expected for each station in gray bars. This was estimated with the division of the coefficients $(a / b)$ of negative exponential model of species accumulation. The observed richness (black line) was estimated with the total species observed in 72 quadrants for each station carnívoro-carroñero, en este caso fue clasificado dentro del grupo carnívoro móvil. En cuanto a las características del tamaño, consideramos que 29 especies conformaron el grupo de macromoluscos y 5 especies en los micromoluscos: Eatoniella nigra, Eatoniella picea, Toledonia parelata, Neolepton concentricum y Lasaea adansoni.

Las especies con mayor frecuencia de ocurrencia fueron M. edulis platensis, P. purpuratus, S. lessonii y $N$. deaurata (Tabla 2). Las especies con menor frecuencia de ocurrencia fueron T. impervia, E. nigra, E. picea y $T$. parelata (Tabla 2). Dentro del ensamble de moluscos, las especies que presentaron mayor abundancia relativa anual fueron los bivalvos sésiles $M$. edulis platensis $(46,6 \%)$ y $P$. purpuratus $(43,81 \%)$. Sin embargo, al considerar solo el grupo de las especies móviles, destacan en abundancia relativa total los gasterópodos L. caliginosa con $42,9 \%$ y S. lessonii $21,8 \%$. Cabe destacar, el hallazgo de $F$. soluta y $T$. impervia, primer reporte de estas especies para el hábitat intermareal de la región de canales y fiordos Magallánicos.

\section{VARIACIÓN ESTACIONAL}

La riqueza de especies presentó cambios significativos entre estaciones (ver Tabla 3), esta diferencia fue observada sólo entre invierno y verano (Riqueza de especies: $P=0,003)$, siendo mayor la riqueza de especies observadas y esperadas en verano que invierno (ver Fig. 2). También durante verano se registró una mayor cantidad de especies de baja frecuencia de ocurrencia $(<$ 2\%), tal como T. atrata, Fissurella cf. picta, F. soluta, A. monodon y $T$. parelata (Tabla 2). Por otro lado, la abundancia de individuos del ensamble de moluscos por cuadrante presentó diferencias significativas entre estaciones (ver Tabla 3), específicamente entre invierno y verano (Abundancia: $P=0,001$ ), pero con mayor número de individuos en invierno (ver Tabla 2). Por ejemplo, la abundancia promedio de invierno fue de $117,9 \pm 15,8$ E.E individuos por $625 \mathrm{~cm}^{2}$, mientras que en verano fue de $90,1 \pm 15,7$ E.E individuos por $625 \mathrm{~cm}^{2}$. Esto principalmente es debido a la gran cantidad de individuos encontrados de M. edulis platensis y principalmente para P. purpuratus (ver Tabla 2).

La estructura de los herbívoros móviles en términos de composición y abundancia presentó diferencia significativas entre las estaciones (ver Tabla 3). El test $a$ posteriori muestra principalmente diferencias entre 
Tabla 3. Análisis de permutaciones (PERMANOVA) para diferentes variables del ensamble de moluscos de bahía Róbalo. El diseño fue de tipo anidado, considerando estación, sectores y nivel intermareales. Los datos se basaron en la disimilaridad de Bray-Curtis, fueron transformados a raíz cuarta y se realizaron $\mathbf{9 9 9}$ permutaciones. Las abreviaciones $\mathrm{F}-\mathrm{S}=$ filtradores sésiles, $\mathrm{H}-\mathrm{M}=$ herbívoros móviles y $\mathrm{C}-\mathrm{M}=$ carnívoros móviles. EI signo asterisco $(*)$ indica diferencias estadísticamente significativas / Analysis of permutations (PERMANOVA) for different variables of molluscs assemblage of Róbalo Bay. The sampling design was nested, considering season, sectors and intertidal level. Data were transformed to the fourth square root without permutation restrictions, based in Bray-Curtis dissimilarity analysis, the number of permutations was of 999. The abbreviations $\mathrm{F}-\mathrm{S}=$ filter-sessile, $\mathrm{H}-\mathrm{M}=$ herbivores-mobile y $\mathrm{C}-\mathrm{M}=$ carnivores-mobile. Asterisk $\left({ }^{*}\right)$ indicates statistically significant differences

\begin{tabular}{|c|c|c|c|c|c|c|c|c|c|c|c|c|c|}
\hline \multirow[t]{2}{*}{ Fuente } & \multirow[t]{2}{*}{$\mathrm{df}$} & \multicolumn{3}{|c|}{ Riqueza } & \multicolumn{3}{|c|}{ Abundancia } & \multicolumn{3}{|c|}{ F-S (3 especies) } & \multicolumn{3}{|c|}{ H-M (11 especies) } \\
\hline & & MS & $\mathrm{F}$ & $P$ & MS & $\mathrm{F}$ & $P$ & MS & $\mathrm{F}$ & $P$ & MS & $\mathrm{F}$ & $P$ \\
\hline Estación & 3 & 4760,6 & 6,395 & $0,001 *$ & 5548,9 & 7,232 & $0,001 *$ & 4926,7 & 4,067 & $0,002 *$ & 9121,04 & 3,448 & $0,002^{*}$ \\
\hline Sectores (Est) & 20 & 6936,4 & 9,318 & $0,001^{*}$ & 7044,03 & 9,181 & $0,001^{*}$ & 7620,9 & 6,292 & $0,001 *$ & 11436,7 & 4,324 & $0,001^{*}$ \\
\hline Nivel (Sec(Est)) & 48 & 610,03 & 0,819 & 0,803 & 1157,9 & 1,509 & $0,019^{*}$ & 1688,5 & 1,394 & 0,015 & 3543,1 & 1,339 & $0,008^{*}$ \\
\hline Residual & 216 & 744,3 & & & 767,2 & & & 1211,2 & & & 2644,6 & & \\
\hline Total & 287 & & & & & & & & & & & & \\
\hline \multirow[t]{2}{*}{ Fuente } & $\mathrm{df}$ & \multicolumn{3}{|c|}{ C-M (5 especies) } & \multicolumn{3}{|c|}{ M. edulis platensis } & \multicolumn{3}{|c|}{ S. lessonii } & & & \\
\hline & & MS & $\mathrm{F}$ & $P$ & MS & $\mathrm{F}$ & $P$ & MS & $\mathrm{F}$ & $P$ & & & \\
\hline Estación & 3 & 2708,3 & 2,148 & 0,081 & 1997,9 & 1,909 & 0,113 & 6353,1 & 3,43 & $0,017^{*}$ & & & \\
\hline Sectores (Est) & 20 & 3742,2 & 2,968 & $0,001 *$ & 7073,9 & 6,761 & $0,001 *$ & 7738,1 & 4,177 & $0,001 *$ & & & \\
\hline Nivel (Sec(Est)) & 48 & 1738,2 & 1,378 & $0,032 *$ & 1402,4 & 1,341 & 0,054 & 2588,9 & 1,397 & 0,06 & & & \\
\hline Residual & 216 & 1260,7 & & & 1046,2 & & & 1852,2 & & & & & \\
\hline Total & 287 & & & & & & & & & & & & \\
\hline
\end{tabular}

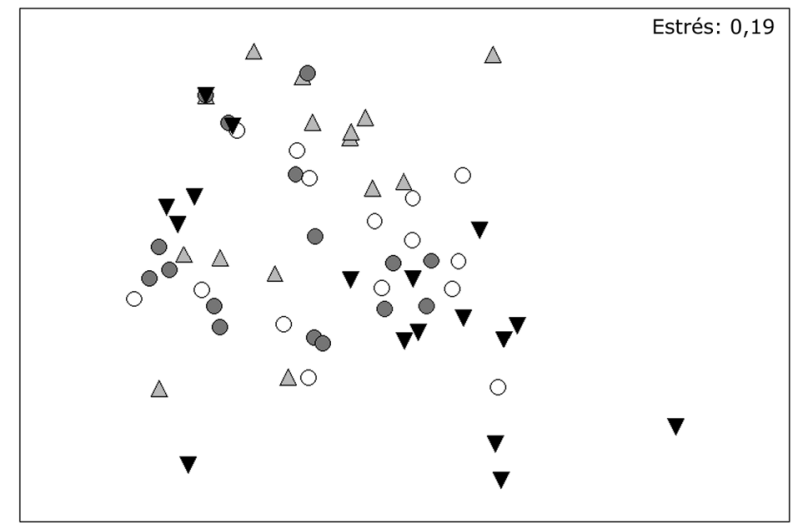

$\triangle$ Otoño Invierno $\bigcirc$ Primavera $\nabla$ Verano

Figura 3. Escalamiento Multidimensional (MDS) para el grupo funcional de los herbívoros móviles. Los datos fueron transformados a raíz cuarta, usando la distancia de disimilaridad de Bray-Curtis / Multi-dimensional Scaling (MDS) for functional group of mobile herbivores. Data were transformed to the fourth square root and was used Bray-Curtis dissimilarity analysis verano-otoño $(P=0,001)$ y en menor medida entre veranoinvierno $(P=0,017)$. Estas diferencias se visualizan en el análisis MDS (Fig. 3), las cuales principalmente se observan entre verano-otoño. Una de las razones de esta diferencia, es el aumento de individuos observados en verano de $L$. caliginosa y especies de la familia Nacellidae (Tabla 2). En cuanto a los carnívoros móviles, no se observaron cambios significativos entre las estaciones. A su vez, la especie más abundante de bahía Róbalo $M$. edulis platensis no presentó una variación significativa entre las estaciones (ver Tabla 3).

\section{VARIACIÓN ESPACIAL HORIZONTAL}

La riqueza de especies y la abundancia de moluscos variaron significativamente entre los sectores intermareales (ver Tabla 3). Esto debido principalmente a que el sector 3 (canto rodado pequeño con influencia de agua dulce) presentó una baja riqueza y abundancia con respecto a los demás sectores. Por ejemplo, el sector 3 tuvo un promedio riqueza de $0,85 \pm 0,14$ especies por cuadrante (Fig. 4) y sólo se registraron 3 especies de moluscos (M. edulis platensis, P. purpuratus y $S$. 
lessonii). En cambio, los demás sectores presentaron un valor promedio $>3$ especies por cuadrante. El valor más alto de riqueza de especies fue observado para sector 1 (canto rodado grande, expuesto al oleaje) con un promedio de 4,2 especies por cuadrante (Fig. 4).

Cabe destacar, que el sector 1 registró la mayor cantidad de especies con baja frecuencia de ocurrencia (i.e., $T$. atrata, T. chilensis, T. geversianus, A. monodon, L. adansoni, N. concentricum, O. marginata y T. parelata). Esta característica en la composición del ensamble es representada por el alto valor de $\mathrm{N} 1$ de la serie de Hill (4,05 especies), con respecto a los demás sectores (Fig. 4). También en el sector 1 se registraron las 9 especies colectadas fuera de los cuadrantes (ver Tabla 2). Por lo tanto, diversidad de este sector intermareal expuesto al oleaje y con grandes bolones fue mucho mayor que los demás sectores del intermareal en bahía Róbalo.
La diversidad a una escala espacial horizontal del intermareal, es decir, considerando los 6 sectores, mostró un valor de diversidad beta alto: 0,83 de acuerdo al índice de Whittaker. Si se excluye del análisis al sector 3, debido a sus contrastantes características de sustrato y cercanía de agua dulce, el índice de diversidad beta de Whittaker DB entre los sustratos 1, 2, 4, 5 y 6 todavía presenta un valor alto: 0,58. Por lo tanto, las variaciones más importantes en el ensamble de moluscos se encontraron en el nivel horizontal asociado a la heterogeneidad de hábitats con distintos sustratos, exposición al oleaje y condiciones de salinidad.

\section{VARIACIÓN ESPACIAL VERTICAL}

La riqueza de especies entre los diferentes niveles intermareales no presentó diferencias significativas (Tabla 3). No obstante, la mayor riqueza fue observada en el

a)

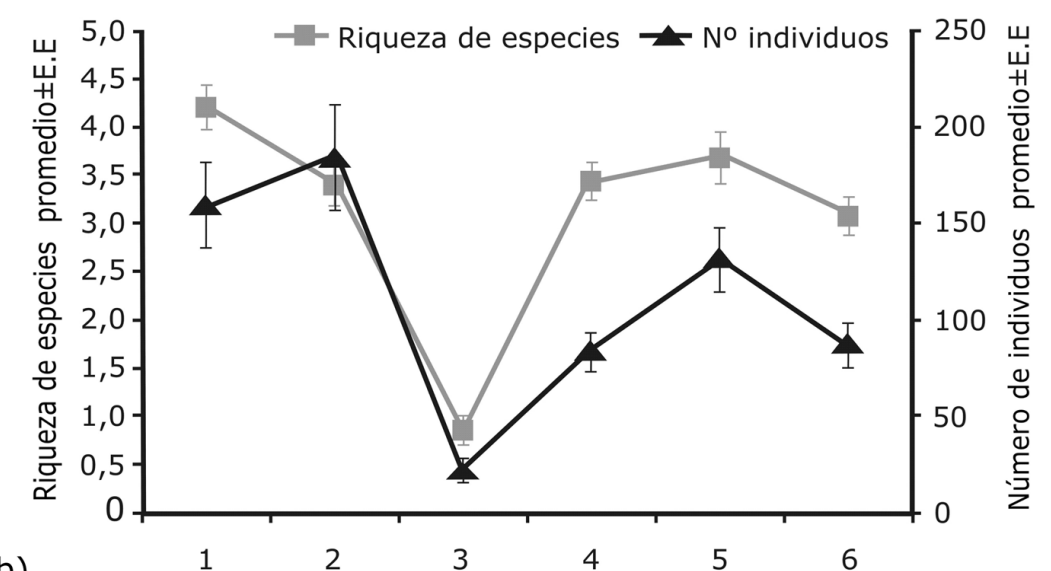

b)

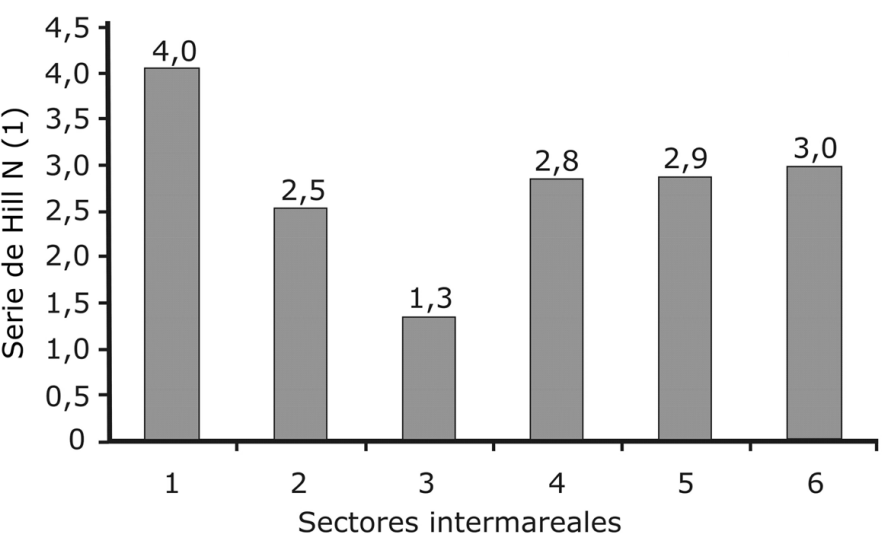

Figura 4. a) Promedio de la riqueza de especies \pm error estándar (E.E) y número de individuos promedio \pm error estándar (E.E) de moluscos por sector intermareal. b) Índice de diversidad alfa de la serie de Hill N (1) de moluscos en sectores intermareales rocosos de bahía Róbalo / a) Average species richness \pm standard error (S.E) and average abundance \pm standard error (S.E). b) Alpha diversity index series Hill $\mathrm{N}$ (1) of rocky intertidal molluscs sectors in Róbalo Bay 
nivel medio del intermareal (Tabla 4). Para la abundancia se observaron cambios significativos entre los diferentes niveles intermareal (Tabla 4), pero estos se detectaron en algunas ocasiones cuando se comparó el nivel alto versus bajo, por ejemplo, durante invierno en el sector 3 ( $P=$ 0,001). En términos específicos, el análisis SIMPER mostró que el grupo funcional de filtradores sésiles como $M$. edulis platensis y $P$. purpuratus no se evidenciaron cambios proporcionales en abundancia y ocurrencia para cada nivel intermareal. Por ejemplo, M. edulis platensis presentó sobre un $60 \%$ de contribución SIMPER y una frecuencia de ocurrencia $>80 \%$ en los cuadrantes para cada nivel intermareal (Tabla 4). En el caso de $P$. purpuratus el porcentaje de contribución SIMPER varió entre $38,7 \%$ en nivel alto, a $27,8 \%$ en el nivel bajo. Con una frecuencia de ocurrencia sobre el $60 \%$ en los cuadrantes para cada nivel intermareal.
El grupo funcional de carnívoros móviles exhibió una baja abundancia con un total de 148 individuos para todo el periodo de estudio. El principal componente en los 3 niveles intermareales fue el carnívoro carroñero $P$. plumbea, este superó el $90 \%$ de contribución para este grupo funcional en todos los niveles, su mayor número total de individuos y frecuencia de ocurrencia fue encontrado en el nivel medio del intermareal con un total de 93 individuos y $26 \%$ de ocurrencia (Tabla 4). La principal diferencia considerando a todos los carnívoros móviles entre niveles intermareales, fue observada entre el nivel alto versus bajo, registrándose un promedio de disimilitud de 43,3\%. Esto debido al aumento de especies en el nivel bajo del intermareal. Cuatro especies conformaron este grupo: T. geversianus, A. monodon, F. soluta y $P$. plumbea.

Tabla 4. Porcentaje de contribución SIM PER $(\% \mathrm{C})$ de especies de moluscos dentro de cada grupo funcional $(\% \mathrm{C})$ para el nivel alto, medio y bajo de intermareal de bahía Róbalo. Para cada especie se determinó el número total de individuos ( ${ }^{\circ}$ ind), frecuencia de ocurrencia (Fr). También se muestra el promedio de individuos y la riqueza promedio de especies \pm error estándar del total ensamble de moluscos por nivel intermareal ( $n=96$ cuadrantes). El signo $(t)$ indica que la especie se presentó en cada nivel intermareal, pero no alcanzó el $90 \%$ de contribución SIMPER para cada grupo funcional de moluscos / Róbalo Bay, Beagle Channel high, medium and low intertidal SIMPER percentage contribution for molluscs species within each functional group $(\% \mathrm{C})$. For each of these specie, we determine individuals' totals $\left(\mathrm{N}^{\circ}\right.$ ind) and occurrence frequency (Fr). The table shows abundance and species richness mean \pm standard error for total assemblage intertidal molluscs level ( $n=96$ quadrants). The sign $(+)$ indicates that the specie are present for intertidal level, but did not reach the $90 \%$ contribution SIMPER for each functional group

\begin{tabular}{|c|c|c|c|c|c|c|c|c|c|}
\hline & \multicolumn{3}{|c|}{ Alto } & \multicolumn{3}{|c|}{ Medio } & \multicolumn{3}{|c|}{ Bajo } \\
\hline & $\mathrm{N}^{\circ}$ ind & $\mathrm{Fr}$ & $\% \mathrm{C}$ & $\mathrm{N}^{\mathrm{o}}$ ind & $\mathrm{Fr}$ & $\% \mathrm{C}$ & $\mathrm{N}^{\mathrm{o}}$ ind & $\mathrm{Fr}$ & $\% \mathrm{C}$ \\
\hline \multicolumn{10}{|l|}{ Filtradores sésiles } \\
\hline M. edulis platensis & 4190 & 82,3 & 60,8 & 5420 & 83,3 & 62,8 & 5331 & 83,3 & 67,5 \\
\hline P. purpuratus & 3145 & 72,9 & 38,7 & 5940 & 70,8 & 36,9 & 4944 & 60,4 & 27,8 \\
\hline \multicolumn{10}{|l|}{ Carnívoro móvil } \\
\hline P. plumbea & 9 & 6,3 & 100 & 93 & 26 & 100 & 31 & 14,6 & 96,6 \\
\hline \multicolumn{10}{|l|}{ Herbívoros móviles } \\
\hline P. aurata & 6 & 4,2 & + & 17 & 11,5 & 3,8 & 20 & 14,6 & + \\
\hline N. magellanica & 18 & 10,4 & + & 22 & 11,5 & + & 50 & 21,9 & 12,2 \\
\hline N. deaurata & 22 & 8,3 & + & 69 & 19,8 & 12,6 & 74 & 17,7 & 7,8 \\
\hline S. ceciliana & 87 & 15,6 & 8,5 & 32 & 10,4 & + & 108 & 14,6 & 6,2 \\
\hline L. caliginosa & 575 & 12,5 & 6,4 & 218 & 10,4 & 3,9 & 127 & 6,3 & + \\
\hline S. lessonii & 89 & 39,6 & 78,5 & 202 & 41,7 & 73,3 & 188 & 39,6 & 64,1 \\
\hline Abundancia (Media \pm E.E) & \multicolumn{3}{|c|}{$90,6 \pm 10,1$} & \multicolumn{3}{|c|}{$126,8 \pm 13,9$} & \multicolumn{3}{|c|}{$116,1 \pm 15,6$} \\
\hline Riqueza de especies (Media \pm E.E) & \multicolumn{3}{|c|}{$2,7 \pm 0,2$} & \multicolumn{3}{|c|}{$3,3 \pm 0,2$} & \multicolumn{3}{|c|}{$3,3 \pm 0,2$} \\
\hline
\end{tabular}


El grupo funcional de herbívoros móviles presenta la mayor riqueza de especies en los niveles medios y bajos: 13 y 12 especies, respectivamente. Cabe destacar, al gasterópodo pulmonado S. lessonii, que presentó la mayor contribución SIMPER al grupo funcional de los herbívoros móviles en todos los niveles intermareales: sobre un $60 \%$ de contribución SIMPER, con frecuencia de ocurrencia $>39 \%$ en cada nivel intermareal (Tabla 4). La principal diferencia para los herbívoros móviles entre diferentes niveles intermareales se presenta para el nivel alto versus bajo con un $70,4 \%$ de disimilitud, esto debido a los cambios de abundancia de las especies. Por ejemplo, L. caliginosa en el nivel alto presentó un total de 575 individuos, mientras que en el nivel bajo disminuyó a un total de 127 individuos (Tabla 4). El caso inverso sucede con $N$. deaurata que presenta en el nivel alto un total 22 individuos, en cambio en el intermareal bajo aumenta a un total de 74 individuos (Tabla 4). Esta variación de menor de abundancia en niveles altos y aumento en niveles intermareales bajos fue observado para otros macroherbívoros como $P$. aurata, N. magallanica, $S$. ceciliana y $O$. marginata.

\section{Discusión}

\section{Caracterización del ensamble de moluscos}

Los estudios de moluscos y su caracterización ecológica de los intermareales rocosos del sistema de canales subantárticos de Magallanes son escasos y restringidos a los meses estivales. Respecto a otros estudios sobre la riqueza de especies en canales, se menciona por ejemplo, que en bahía Laredo se han documentado 26 especies (Mutschke et al. 1998), canal Whiteside 17 especies (Rios \& Mutschke 1999) y en la boca oriental de estrecho de Magallanes (Buque Quemado) 12 especies (Aldea \& Rosenfeld 2011).

El presente trabajo documenta números de especies similares a los reportados en otros estudios anteriores, esto si se consideran los números de especies por cada estación de muestreo: 14 en otoño, 16 en invierno, 21 en primavera y 24 en verano. Sin embargo, si se suman los números de especies de las 4 estaciones el número aumenta a 34 especies. Esto representa una mayor riqueza específica de moluscos reportados en un sitio de estudio intermareal en el sistema de canales y fiordos subantárticos, considerando que el esfuerzo de muestreo se desarrolló sólo en un sitio (bahía Róbalo), pero con alta intensidad de muestreo temporal. Estudios comunitarios de la evaluación in situ de la biodiversidad en forma sistemática a largo plazo con una alta resolución taxonómica son fundamentales para realizar comparaciones locales o regionales (e.g., Broitman et al. 2011). La identificación de zonas de alta diversidad de moluscos y otros organismos son relevantes para la aplicación de metodologías de conservación marina (Fernández et al. 2000). En este contexto, esta investigación malacológica confirma el valor para la conservación biológica del borde costero de isla Navarino en la Reserva de Biósfera Cabo de Hornos.

Las especies de moluscos más abundantes de bahía Róbalo fueron los filtradores sésiles M. edulis platensis y $P$. purpuratus. Esta abundancia y plasticidad ecológica es reconocida dentro de los canales, ya que habitan en diferentes niveles intermareales (Benedetti-Cecchi \& Cinelli 1997) y en zonas de baja y alta salinidad (ver Davenport 1995, Soto et al. 2012). Por otro lado, para los moluscos carnívoros la mayor abundancia correspondió a $P$. plumbea, una especie endémica de la provincia biogeográfica Magallánica (Aldea \& Rosenfeld 2011), considerada de hábitos carroñeros en la zona intermareal (Mutschke et al. 1998). En bahía Róbalo P. plumbea registró su mayor abundancia en la zona media del intermareal, asociado también a matrices de $P$. purpuratus y $M$. edulis platensis, incluso en ocasiones se registraron oviposturas bajo las rocas (J. Ojeda. obs. pers). Las especies de moluscos carnívoros de menor abundancia y frecuencia fueron $T$. impervia y $F$. soluta.

Cabe destacar una descripción sobre el estado taxonómico de la especie $F$. soluta perteneciente a la familia Naticidae, esto porque en la actualidad es considerada como sinónimo junior de $F$. patagonica (ver Bouchet 2014). No obstante, Bouchet (2014) se basó en el trabajo de Torigoe \& Inaba (2011) para actualizar la sistemática de este grupo. Sin embargo, estos últimos autores al realizar una revisión y clasificación de la familia Naticidae no citaron en su trabajo taxonómico el realizado por Pastorino (2005), sobre la familia Naticidae de Patagonia (Torigoe \& Inaba 2011). En este trabajo Pastorino (2005) comenta que F. soluta y Euspira patagonica Philippi, 1845, son 2 especies distintas, ya que E. patagonica presenta caracteres de la concha y la estructura de la rádula diferente a $F$. soluta (ver Pastorino 2005, página 236). Por lo tanto, en la actualidad ambas especies deberían ser consideradas como válidas, pero están siendo consideradas como sinónimo junior de $F$. patagonica (Bouchet 2014). Futuras investigaciones utilizando herramientas moleculares podrán dilucidar, si efectivamente estas especies son genéticamente diferentes. 
Finalmente, F. soluta como T. impervia constituyen el primer reporte de presencia de las especies en la zona intermareal en la región de canales y fiordos subantárticos. Previamente ha sido considerada principalmente de hábitos submareales llegando incluso bajo los $100 \mathrm{~m}$ de profundidad (Pastorino 2005).

Los macroherbívoros móviles presentan una alta riqueza en bahía Róbalo, las especies más abundantes y frecuentes fueron S. lessonii y L. caliginosa. A su vez, una de las particularidades de la composición de moluscos en bahía Róbalo y en las demás zonas costeras de los canales subantárticos es el nivel de endemismo de los macroherbívoros como $K$. lateralis, L. caliginosa y $N$. flammea, que también ha sido reconocida en otros estudios (e.g., Guzmán 1978, Ríos \& Guzmán 1982, Ríos \& Mutschke 1999, Aldea \& Rosenfeld 2011). Este carácter endémico del ensamble de moluscos requiere una mayor profundidad sobre aspectos ecológicos que consideren la estructura y procesos funcionales de las especies. En zonas más septentrionales, posterior al quiebre biogeográfico de la provincia Magallánica $42^{\circ} \mathrm{S}-46^{\circ} \mathrm{S}$ (Reid \& Osorio 2000), en las costas expuesta del sur de Chile (e.g., Mehuín: 39²4'S; 73²13'W), uno de los macroherbívoros más abundantes es $S$. lessonii y en menor medida especies como Fissurella cf. picta, Chiton granosus, Tegula atra y Prisogaster niger (Jara \& Moreno 1984). Por otro lado, antecedentes en la costa Atlántica de Argentina (38³4'S; 58³8'W), muestran que $S$. lessonii es el herbívoro más abundante en la zona media de intermareal (Adami 2008). Por lo tanto, este gasterópodo pulmonado es una de las especies más frecuentes y abundante en las costas rocosas al sur de Sudamérica.

\section{VARIACIONES ESTACIONALES}

La variabilidad estacional de la radiación fotosintéticamente activa (Luz PAR) y el fotoperiodo para la zona de canales subantárticos probablemente es un factor relevante en la estructuración de las comunidades intermareales. Por ejemplo, las macroalgas intermareales en bahía Róbalo presentan una variación estacional del ensamble en biomasa (gramos de peso húmedo en $625 \mathrm{~cm}^{2}$ ) entre invierno y verano, con un promedio de $21,3 \pm 5,2 \mathrm{~g}$ y $69,5 \pm 8,6 \mathrm{~g}$, respectivamente (Ojeda 2012). Este cambio estacional también es similar para la riqueza de taxa de macroalgas (Ojeda 2012). En el caso del presente estudio, los moluscos mostraron un patrón similar para el ensamble de herbívoros móviles, específicamente para L. caliginosa y las especies de la familia Nacellidae. Este tipo de variación estacional debe ser abordado desde otros puntos ecológicos como los cambios de peso corporal o hábitos tróficos. Por ejemplo, Harley (2001) en costas templadas del hemisferio norte ha registrado variaciones en el peso corporal de los individuos, donde el efecto sombra durante invierno en gasterópodos herbívoros produce una disminución de su biomasa. En el caso de los hábitos tróficos se ha identificado que las macroalgas no son la única oferta de alimento potencial para los herbívoros móviles, ya que el perifiton o 'biofilms' constituye otra fuente de energía disponible en el intermareal (e.g., Aguilera et al. 2013). Para el caso de $S$. lessonii, se ha observado experimentalmente que se alimenta de perifiton e incluso su proceso de pastoreo tiene efectos positivos en la diversidad del perifiton intermareal (Aguilera et al. 2013).

Una de las especies abundantes de herbívoros es $N$. deaurata que presentó un claro aumento en abundancia durante estaciones estivales con respecto a estaciones invernales en bahía Róbalo. Este tipo de variación también fue documentada por Andrade (2009), en la zona de bahía Laredo (estrecho de Magallanes). Probablemente este cambio está asociado a la movilidad de $N$. deaurata a zonas submareales someras, a pesar de ser una especie del intermareal bajo (Morriconi \& Calvo 1993), también ha sido documentada para hábitat submareales como bosques de kelps (Castilla 1985, Adami \& Gordillo 1999). Casos sobre el desplazamiento en este género, han sido reportados en la Antártica para Nacella concinna en las islas Shetland del sur, donde se describe que durante invierno habita preferentemente la zona sublitoral y en verano se desplaza hacia el intermareal (Kim 2001). Finalmente, es necesario indagar de forma más específica las interacciones ecológicas alga-herbívoro, estudios experimentales ayudarán a entender los hábitos estacionales de los herbívoros móviles en altas latitudes como los canales y fiordos subantárticos.

En contraste a la esta dinámica estacional de herbívoros móviles, se observó que los filtradores sésiles no presentaban un aumento hacia épocas estivales. Por ejemplo, en $M$. edulis platensis no presentó una variación significativa en la abundancia entre estaciones e incluso $P$. purpuratus su mayor abundancia se observó durante invierno. Este tipo de estabilidad en la abundancia de las matrices de M. edulis platensis había sido descrito para la zona del estrecho de Magallanes por Langley et al. (1980). Otro tipo de antecedentes sobre la estacionalidad de los filtradores sésiles ha sido entregado desde la arqueología en zonas intermareales del canal Beagle. Por 
ejemplo, se describe que el aporte nutricional de $M$. edulis platensis, en $100 \mathrm{~g}$ de carne deshidratada, contiene 65,8 $\mathrm{g}$ de proteínas y produce en total alrededor de $350 \mathrm{kcal}$, con una mínima variación estacional en el rendimiento alimenticio individual, es decir, la variabilidad entre otoño, primavera y verano en cuanto a proteínas o kilocalorías es relativamente baja (Orquera 2000).

\section{VARIACIÓN ESPACIAL HORIZONTAL}

El presente estudio reporta marcada influencia de la heterogeneidad de hábitats con distintos sustratos, exposición al oleaje e influencia de agua dulce sobre la diversidad y abundancia de moluscos en la eco-región subantártica de Magallanes. En bahía Róbalo se detectaron diferencias de un orden de magnitud entre sectores con más de 150 individuos por cuadrantes en sectores 1 y 2, a menos de 15 individuos por cuadrante en el sector 3. La riqueza promedio de especies también fue 3 veces mayor en el sector 1 respecto al sector 3 (ver Fig. 4). Como señalamos anteriormente, el sector 1 no solo presentó un mayor de especies conspicuas, sino también de especies de baja detección como los micromoluscos L. adansoni, $N$. concentricum y $T$. parelata. Estas características de estructuración del ensamble no fueron observadas para el resto de los sectores intermareales de bahía Róbalo.

La variabilidad espacial de los ensambles costeros en la zona de canales, es reconocida por las diferencias de estructuración de especies conspicuas en diferentes niveles intermareales (e.g., Benedetti-Cecchi \& Cinelli 1997) y también ha sido caracterizada por la diversidad de biotopos intermareales asociados a una heterogeneidad espacial (Soto et al. 2012). Por lo tanto, los hábitats intermareales parecen tener un alto grado de cambio o reemplazo de especies, es decir, diversidad beta. Benedetti-Cecchi \& Cinelli (1997), mencionan que la variabilidad en la distribución de las especies podría ser causada por procesos ecológicos heterogéneos, como las diferencias espaciales de reclutamiento, tolerancia al estrés físico, depredación y competición. Koleff (2005) menciona que gran parte de los trabajos ecológicos se han centrado en evaluar la diversidad alfa, realizando comparaciones locales o regionales. En el sistema de canales y fiordos principalmente se han realizado estudios que detallan la riqueza de especies (diversidad alfa) (e.g., Mutschke et al. 1998, Ríos \& Mutschke 1999, Aldea \& Rosenfeld 2011, Rosenfeld et al. 2013). En el presente estudio se ha abordado además el análisis del recambio espacial en la composición de especies del ensamble de moluscos, documentando valores altos de diversidad beta dentro de la bahía Róbalo. Estos resultados de una alta diversidad beta, sugieren la relevancia de investigar la diversidad de moluscos considerando la diversidad de hábitats al interior de las bahías y probablemente entre bahías en la eco-región subantártica de Magallanes. La heterogeneidad ambiental tanto de los factores físicos como biológicos de las zonas intermareales de altas latitudes como el sistema de canales y fiordos probablemente facilita la alta diversidad alfa y beta de moluscos en las costas rocosas.

\section{VARIACIÓN ESPACIAL VERTICAL}

Respecto a los patrones de riqueza de especies y abundancia de moluscos en el eje vertical del intermareal, nuestros resultados son similares a los reportados por otros estudios (e.g., Benedetti-Cecchi \& Cinelli 1997, Mutschke et al. 1998, Ríos \& Mutschke 1999). La especie más conspicua como M. edulis platensis no presentó un patrón definido de distribución. Este tipo de estructuración a escala vertical-horizontal, también fue descrito por Benedetti-Cecchi \& Cinelli (1997) para diferentes costas en el estrecho de Magallanes, considerando como metodología de muestreo la cobertura. Por otro lado, para especies móviles conspicuas se observaron agrupamientos asociados a los niveles intermareales. Por ejemplo, en L. caliginosa se observó con una mayor abundancia en el intermareal alto, mientras que $P$. plumbea lo fue en la zona media y $N$. deaurata en el intermareal bajo de bahía Róbalo. Este tipo de zonación intermareal también ha sido documentada de forma descriptiva para bahía Laredo (estrecho de Magallanes) (ver Mutschke et al. 1998).

Finalmente, el presente trabajo entrega una de las primeras caracterizaciones estacionales para ensambles intermareales rocosos de la Reserva de Biósfera Cabo de Hornos, en donde se observan cambios estacionales tanto en la riqueza de especies como en la composición y abundancia de herbívoros móviles. Por el contrario, la especies como M. edulis platensis y el ensamble de carnívoros móviles muestran una abundancia estable durante las estaciones del año. En términos espaciales es posible observar una alta diversidad beta de moluscos, asociada a la variabilidad espacial. Estos cambios se pueden detectar entre sitios separados por centenas de metros, donde la heterogeneidad ambiental local probablemente juega un rol ecológico en la estructuración de ensambles y comunidades en los canales y fiordos subantárticos del sur de Chile. 


\section{Agradecimientos}

Al apoyo logístico brindado por la Universidad de Magallanes sede Puerto Williams representados por Dr. Francisca Massardo, MSc. Ximena Arango, MSc. Cristóbal Pizarro, Tamara Contador y Rosaria Civitelli. J. Ojeda agradece al Instituto de Ecología y Biodiversidad (IEB) por la beca otorgada del proyecto ICM, código P05002. S. Rosenfeld agradece al IEB por la beca ICM, código P05-002. J. Marambio agradece al IEB financiamiento BASAL, código PFB -23-2008. Gracias por los comentarios a esta investigación a Dr. Jorge Terrados, Dr. Jaime Rau y Dr. Marcelo Rivadeneira. Finalmente gracias al Magister en Ciencias con Mención en Manejo y Conservación de Recursos Naturales de Ambientes Subantárticos de la Universidad de Magallanes.

\section{LITERATURA CITADA}

Adami ML. 2008. Efectos de la herbivoría de la lapa Siphonaria lessoni Blainville, 1824 (Gastropoda) sobre la comunidad asociada a Brachidontes rodriguezii (d' Orbigny, 1846) (Bivalvia). Revista de Museo Argentino de Ciencias Naturales, Argentina 10: 309-317.

Adami ML \& S Gordillo. 1999. Structure and dynamics of the biota associated with Macrocystis pyrifera (Phaeophyta) from the Beagle Channel, Tierra del Fuego. Scientia Marina 63(Suppl 1): 183-191.

Aguilera MA, SA Navarrete \& BR Broitman. 2013. Differential effects of grazer species on periphyton of a temperate rocky shore. Marine Ecology Progress Series 484: 63-78.

Aldea C \& S Rosenfeld. 2011. Moluscos intermareales de la Playa Buque Quemado (Estrecho de Magallanes, Chile). Revista de Biología Marina y Oceanografía 46: 115-124.

Anderson MJ. 2001. A new method for non-parametric multivariate analysis of variance. Austral Ecology 26: 32-46.

Anderson MJ. 2005. PERMANOVA: a FORTRAN computer program for permutational multivariate analysis of variance, 24 pp. Department of Statistics, University of Auckland.

Andrade C. 2009. Estructura trófica del ensamble de moluscos en el intermareal de bloques y cantos (Bahía Laredo, estrecho de Magallanes). Tesis de Magister, Facultad de Ciencias, Universidad de Magallanes, Punta Arenas, 123 pp.

Aranzamendi MC, CN Gardenal, JP Martin \& R Bastida. 2009. Limpets of the genus Nacella (Patellogastropoda) from the Southwestern Atlantic: species identification based on molecular data. Journal of Molluscan Studies 75: 241-251.

Benedetti-Cecchi L \& F Cinelli. 1997. Spatial distribution of algae and invertebrates in the rocky intertidal zone of the Strait of Magellan: are patterns general? Polar Biology 18 : 337-343.
Bouchet P. 2014. Falsilunatia patagonica. In: WoRMS, World Register of Marine Species. Vlaams Instituut voor de Zee, VLIZ, Oostende. <http://www.marinespecies.org/ aphia.php? $\mathrm{p}=$ taxdetails\&id=584317>

Broitman BR, F Véliz, T Manzur, EA Wieters, GR Finke, PA Fornes, N Valdivia \& SA Navarrete. 2011. Geographic variation in diversity of wave exposed rocky intertidal communities along central Chile. Revista Chilena de Historia Natural 84: 143-154.

Camus PA. 2001. Biogeografía marina de Chile continental. Revista Chilena de Historia Natural 74: 587-617.

Camus PA, YY Cid, L Cisterna \& CW Cáceres. 2009. Consumption and digestion of animal food by rocky intertidal herbivores: an evaluation of digestive flexibility and omnivory in three grazing species. Latin American Journal of Aquatic Research 37(2): 191-197.

Camus PA, PA Arancibia \& MI Ávila-Thieme. 2013. A trophic characterization of intertidal consumers on Chilean rocky shores. Revista de Biología Marina y Oceanografía 48: 431-450.

Cárdenas J \& S Gordillo. 2009. Paleoenvironmental interpretation of late Quaternary molluscan assemblages from southern South America: A taphonomic comparison between the Strait of Magellan and the Beagle Channel. Andean Geology 36: 81-93.

Cárdenas J, C Aldea \& C Valdovinos. 2008. Chilean marine mollusca of northern Patagonia collected during the CIMAR10 Fjords cruise. Gayana 72: 202-240.

Castilla JC. 1985. Food webs and functional aspects of the kelp, Macrocystis pyrifera, community in the Beagle Channel, Chile. In: Siegfried WR, PR Condy \& RM Laws (eds). Antarctic nutrient cycles and food webs, pp. 407414. Springer, Berlin, Heidelberg.

Castilla JC \& LR Durán. 1985. Human exclusion from the rocky intertidal zone of central Chile: The effects on Concholepas concholepas (Gastropoda). Oikos 45:391-399.

Clarke KR. 1993. Non-parametric multivariate analysis of changes in community structure. Australian Journal of Ecology 18: 117-143.

Davenport J. 1995. The marine ecology of the Laguna SanRafael (southern Chile) - ice scour and opportunism. Estuarine Coastal and Shelf Science 41: 21-37.

Dayton PK. 1985. The structure and regulation of some south American Kelp Communities. Ecological Monographs 55: 447-468.

Dell RK. 1964. Antarctic and sub-Antarctic Mollusca: Amphineura, Scaphopoda and Bivalvia. Discovery Reports 33: 99-250.

Dell RK. 1990. Antarctic Mollusca with special reference to the fauna of the Ross Sea. Bulletin of the Royal Society of New Zealand 27: 1-311. 
Fernández M, E Jaramillo, PA Marquet, CA Moreno, SA Navarrete, FP Ojeda, CR Valdovinos \& JA Vásquez. 2000. Diversity, dynamics and biogeography of Chilean benthic nearshore ecosystems: an overview and guidelines for conservation. Revista Chilena de Historia Natural 73: 797-830.

Foster MS. 1990. Organization of macroalgal assemblages in the Northeast Pacific: the assumption of homogeneity and the illusion of generality. Hydrobiologia 192: 21-33.

Geiger DL, BA Marshall, WF Ponder, T Sasaki \& A Warén. 2010. Techniques for collecting, handling, preparing, storing and examining small molluscan specimens. Molluscan Research 27: 1-50.

González-Wevar CA, T Nakano, JI Cañete \& E Poulin. 2011. Concerted genetic, morphological and ecological diversification in Nacella limpets in the Magellanic Province. Molecular Ecology 20: 1936-1951.

Gunnarsson K \& A Ingólfsonn. 1995. Seasonal changes in the abundance of intertidal algae in Southwestern Iceland. Botanica Marina 38: 69-77.

Gutiérrez JL, CG Jones, DL Strayer \& OO Iribarne. 2003. Mollusks as ecosystem engineers: the role of shell production in aquatic habitats. Oikos 101: 79-90.

Guzmán L. 1978. Patrón de distribución espacial y densidad de Nacella magallanica (Gmelin, 1971) en el intermareal del sector oriental del estrecho de Magallanes (Mollusca, Gastropoda). Anales del Instituto de la Patagonia, Serie Ciencias Naturales 9: 207-219.

Harley CDG. 2002. Light availability indirectly limits herbivore growth and abundance in a high rocky intertidal community during the winter. Limnology and Oceanography 47: 1217-1222.

Harrelson CC, CL Rawlins \& JP Potyondy. 1994. Stream channel references sites: an illustrated guide to Weld technique. General Technical Report RM-245: 1-63. USDA Forestry Service, Fort Collins, Colo.

Hill MO. 1973. Diversity and evenness: a unifying notation and its consequences. Ecology 54: 427-432.

Hulton NRJ, RS Purves, RD Mcculloch, DE Sugden \& MJ Bentley. 2002. The Last Glacial Maximum and deglaciation in southern South America. Quaternary Science Reviews 21: 233-241.

Jara HF \& CA Moreno. 1984. Herbivory and structure in a midlittoral rocky community: A case in Southern Chile. Ecology 65: 28-38.

Jiménez-Valverde A \& J Hortal .2003. La curva de acumulación de especies y la necesidad de evaluar los inventarios biológicos. Revista Ibérica de Aracnología 8: 15-161.

Kiel S \& SN Nielsen. 2010. Quaternary origin of the inverse latitudinal diversity gradient among southern Chilean mollusks. Geology 38: 955-958.

Kim D. 2001. Seasonality of marine algae and grazers of an Antarctic rocky intertidal, with emphasis on the role of the limpet Nacella concinna Strebel (Gastropoda: Patellidae). Berichte zur Polar und Meeresforschung 397: 1-136.
Koleff P. 2005. Conceptos y medidas de la diversidad beta. En: Halffter G, J Soberón, P Koleff \& A Melic (eds). Sobre la diversidad biológica: el significado de las diversidades Alfa, Beta, Gamma, pp. 19-40. Monografías Tercer Milenio, SEA/ CONABIO/ DIVERSITAS/ CONACYT, Zaragoza.

Kruskal JB \& M Wish. 1978. Multidimensional scaling, 99 pp. Sage Publications, Beverly Hills.

Langley S, L Guzman \& C Ríos. 1980. Aspectos dinámicos de Mytilus chilensis (Hupé, 1840) en el estrecho de Magallanes. I. Distribución, densidad y disposición espacial en el intermareal. Anales del Instituto de la Patagonia, Serie Ciencias Naturales 11: 319-332.

Lewis JR. 1972. The ecology of rocky shores, 323 pp. The English Universities Press, London.

Linse K. 1999. Mollusca of the Magellan region. A cheklist of the species and their distribution. Scientia Marina 63 (Suppl. 1): $399-407$.

Linse K. 2002. The shelled magellanic Mollusca: with special reference to biogeographic relations in the Southern Ocean. Theses Zoologicae 34: 1-252.

Moreno CA, JP Sutherland \& HF Jara. 1984. Man as a predator in the intertidal zone of Southern Chile. Oikos 42: 155-160.

Morriconi E \& J Calvo. 1993. Influencia ambiental sobre el crecimiento alométrico de la valva en Nacella (Patinigera) deaurata (Gmelin, 1791) del Canal Beagle, Argentina. Malacologia 35: 135-140.

Mutschke E, C Ríos \& A Montiel. 1998. Situación actual de la macrofauna presente en el intermareal de bloques y cantos de Bahía Laredo, Estrecho de Magallanes. Anales del Instituto de la Patagonia, Serie Ciencias Naturales 26: 5-29.

Ojeda J. 2012. Dinámica estacional de macroalgas y moluscos intermareales y su relación con el conocimiento tradicional ecológico yagán, en canales subantárticos del Cabo de Hornos: Una aproximación biocultural desde la filosofía ambiental de campo. Tesis de Magister, Facultad de Ciencias, Universidad de Magallanes, Punta Arenas, 144 pp.

Ojeda J, T Contador, S Rosenfeld, CB Anderson, A Mansilla \& JH Kennedy. 2010. Guía para la identificación de los invertebrados marinos y dulceacuícolas de la Reserva de Biósfera Cabo de Hornos, 80 pp. Ediciones Universidad de Magallanes, Punta Arenas.

Orquera LA. 2000. El consumo de moluscos por los canoeros de extremo sur. Relaciones de la Sociedad de Argentina de Antropología, Buenos Aires 24: 307-327.

Pastorino G. 2005. Recent Naticidae (Mollusca: Gastropoda) from the Patagonian Coast. The Veliger 47: 225-258.

Ponder WF \& TM Worsfold. 1994. A review of the rissoiform gastropods of Southwestern South America (Mollusca, Gastropoda). Contributions in Science (Natural History Museum of Los Angeles County) 445: 1-63.

Powell AWB. 1951. Antarctic and Subantarctic Mollusca: Pelecypoda and Gastropoda. Discovery Reports 26: 47196. 
Raffaelli D \& S Hawkins. 1996. Intertidal ecology, 356 pp. Chapman \& Hall, London.

Rau J, C Zuleta, A Gantz, F Saiz, A Cortes, L Yates, AE Spotorno \& E Couve. 1998. Biodiversidad de artrópodos y vertebrados terrestres del Norte Grande de Chile. Revista Chilena de Historia Natural 71: 527-554.

Reid DG \& C Osorio. 2000. The shallow-water marine mollusca of the Estero Elefantes and Laguna San Rafael, southern Chile. Bulletin of the Natural History Museum of London, Zoology 66: 109-146.

Ríos C \& L Guzmán. 1982. Reevaluación de la estructura de la comunidad en una playa de bloques y cantos de alta latitud (Archipielago del Cabo de Hornos). Anales del Instituto de la Patagonia, Serie Ciencias Naturales 13: 213-224.

Ríos C \& E Mutschke. 1999. Community structure of intertidal boulder-cobble fields in the Straits of Magellan, Chile. Scientia Marina 63(Suppl. 1): 193-201.

Rochebrune AT \& J Mabille. 1889. Mollusques. Mision Scientifique du Cap Horn, 129 pp. VI, Zoologie, Paris.

Rosenfeld S \& C Aldea. 2010. Onchidella marginata (Couthouy en Gould, 1952) antecedentes de la especie. Amici Molluscarum, Chile 18: 35-37.

Rosenfeld S \& C Aldea. 2011. An unknown Opisthobranch (Mollusca: Gastropoda) in the Magellan region (Toledonia parelata Dell, 1990): New records and similar species. Anales del Instituto de la Patagonia, Serie Ciencias Naturales 39: 133-136.

Rosenfeld S, J Marambio \& C Aldea. 2013. Comparación de ensambles de moluscos en dos sustratos intermareales de la cuenca central del estrecho de Magallanes. Amici Moluscarum 21(2): 7-18.

Rozzi R, F Massardo, A Mansilla, CB Anderson, A Berghofer, MO Mansilla, MR Gallardo, J Plana, U Berghofer, $X$ Arango, S Russell, PAraya \& E Barros. 2007. La Reserva de Biosfera Cabo de Hornos: un desafío para la conservación de la biodiversidad e implementación del desarrollo sustentable en el extremo austral de América. Anales del Instituto de la Patagonia 35: 55-70.

Rozzi R, JJ Armesto, J Gutierrez, F Massardo, G Likens, CB Anderson, A Poole, $\mathrm{K}$ Moses, G Hargrove, A Mansilla, JH Kennedy, M Willson, K Jax, C Jones, JB Callicott \& MT Kalin. 2012. Integrating ecology and environmental ethics: Earth stewardship in the southern end of the Americas. BioScience 62: 226-236.

Santana A, C Porter, N Butorovic \& C Olave. 2006. Primeros antecedentes climatológicos de Estaciones Automáticas (AWS) en el canal Beagle, Magallanes, Chile. Anales del Instituto de la Patagonia, Serie Ciencias Naturales 34: 5-20.

Schwabe E, G Försterra, V Häusserman, RR Melzer \& M Schrödl. 2006. Chitons (Mollusca: Polyplacophora) from the southern Chilean Comau Fjord, with reinstatement of Tonicia calbucensis Plate, 1897. Zootaxa 1341: 1-27.
Scrosati R \& C Heaven. 2007. Spatial trends in community richness, diversity, and evenness across rocky intertidal environmental stress gradients in eastern Canada. Marine Ecology Progress Series 343: 1-14.

Silva N \& C Calvete. 2002. Características oceanográficas físicas y químicas de canales australes chilenos entre el golfo de Penas y el Estrecho de Magallanes (Crucero CIMAR-FIORDOS 2). Ciencia y Tecnología del Mar, Chile 25: 23-88.

Sirenko B. 2006. Report on the present state of our knowledge with regard to the chitons (Mollusca: Polyplacophora) of the Magellan Strait and Falkland Islands. Venus 65(1-2): 81-89.

Soberón J \& J Llorente. 1993. The use of the species accumulation functions for the prediction of species richness. Conservation Biology 7: 480-488.

Soto E, P Báez, ME Ramírez, S Letelier, J Naretto \& A Rebolledo. 2012. Biotopos marinos intermareales entre Canal Trinidad y Canal Smyth, Sur de Chile. Revista de Biología Marina y Oceanografía 47: 177-191.

Stephenson TA \& A Stephenson. 1949. The universal feature of zonation between tide-marks on rocky coasts. Journal Ecology 37: 289-305.

Strebel H. 1905. Beiträge zur Kenntnis der Molluskenfauna der Magalhaen-Provinz, II. Die Trochiden. Zoologische Jahrbücher, Suppl. 8: 121-166.

Stuardo J. 1964. Distribución de los moluscos marinos litorales en Latinoamérica. Boletín del Instituto de Biología Marina 7: 79-91.

Torigoe K \& A Inaba. 2011. Revision on the classification of recent Naticidae. Bulletin of the Nishinomiya Shell Museum 7: 1-133.

Valdovinos C. 1999. Biodiversidad de moluscos chilenos: Base de datos taxonómica y distribución. Gayana 63: 111-164.

Valdovinos C, SA Navarrete \& PA Marquet. 2003. Mollusk species diversity in the Southeastern Pacific: Why are there more species towards the pole? Ecography 26: 139-144.

Waller CL. 2008. Variability in intertidal communities along a latitudinal gradient in the Southern Ocean. Polar Biology 31: 809-816.

Walozeck D. 1984. Variabilität, Taxonomie und Verbreitung von Chlamys patagonica (King and Broderip, 1832) und Anmerkungen zu weiteren Chlamys-Arten von der Südspitze Süd-Amerikas (Mollusca, Bivalvia, Pectinidae). Verhandlungen des Naturwissenschaftlichen Vereins inHamburg 27: 207-276.

Whittaker RH. 1972. Evolution and measurement of species diversity. Taxon 21: 213-251.

Zelaya D \& C Ituarte. 2004. The genus Neolepton Monterosato, 1875 in Southern South America (Bivalvia: Neoleptonidae). Journal of Molluscan Studies 70: 123-137. 